\title{
Low-cost chemical gas sensors for selective formaldehyde quantification at ppb-level in the field
}

\author{
Manuel Bastuck ${ }^{1,2}$, Donatella Puglisi ${ }^{2}$, Peter Möller ${ }^{2}$, Wolfhard Reimringer ${ }^{3}$, \\ Andreas Schütze ${ }^{1}$, Anita Lloyd Spetz ${ }^{2}$, Mike Andersson ${ }^{2,4}$ \\ ${ }^{1}$ Lab for Measurement Technology, Saarland University, Saarbrücken, Germany \\ ${ }^{2}$ Sensors and Actuator Systems, Dept. Physics, Chemistry and Biology, Linköping University, \\ Linköping, Sweden \\ ${ }^{3} 3 \mathrm{~S} \mathrm{GmbH}$, Saarbrücken, Germany \\ ${ }^{4}$ SenSiC AB, Kista, Sweden \\ m.bastuck@Imt.uni-saarland.de
}

\begin{abstract}
Data from a silicon carbide based field-effect transistor were recorded over a period of nine days in a ventilated school room. For enhanced sensitivity and selectivity especially to formaldehyde, porous iridium on pulsed laser deposited tungsten trioxide was used as sensitive layer, in combination with temperature cycled operation and subsequent multivariate data processing techniques. The sensor signal was compared to reference measurements for formaldehyde concentration, $\mathrm{CO}_{2}$ concentration, temperature, and relative humidity. The results show a distinct pattern for the reference formaldehyde concentration, arising from the day/night cycle. Taking this into account, the projections of both principal component analysis and partial least squares regression lead to almost the same result concerning correlation to the reference. The sensor shows cross-sensitivity to an unidentified component of human activity, presumably breath, and, possibly, to other compounds appearing together with formaldehyde in indoor air. Nevertheless, the sensor is able to detect and partially quantify formaldehyde below $40 \mathrm{ppb}$ with a correlation to the reference of 0.48 and negligible interference from ambient temperature or relative humidity.
\end{abstract}

Key words: SiC-FET, multivariate data processing, indoor air quality, VOC, sick building syndrome

\section{Introduction}

Buildings account for a large amount of the total energy consumption [1]. Hence, optimization for energy efficiency in this field can lead to significant reductions of costs, energy dependency and greenhouse gases. The European Directive 2010/31/EU [1] requires the member states to build only low-energy buildings after 2019 (public) and 2020 (private). Among others measures, airtightness plays an important role as heat loss through convection negatively influences the energy footprint, leading to a trend towards tighter buildings [2]. The reduced ventilation must be accounted for by installing ventilation systems which allow for a more controlled air flow and a healthy and comfortable indoor climate, which is important given that humans spend more than $85 \%$ of their time indoors [3].

The best compromise between energy consumption and good indoor air quality (IAQ) can be reached with automatic ventilation control. A definition for the controlled variable, i.e. indoor air quality, is, however, not easy to find. Some factors affect human wellness immediately, like humidity or carbon dioxide $\left(\mathrm{CO}_{2}\right)$ [4]. Another large group of substances affecting IAQ are volatile organic compounds (VOCs). There is a wide variety of those substances, which is why they are most often reported as sum (of masses or volume) of all measured compounds (total VOC, TVOC) [5]. However, this does not account for very different risk potentials of those various substances ranging from no effect on human health, to headaches and even cancer after long-term exposure to typical indoor concentrations. To target only hazardous substances, selective detection of one compound or at least a group of compounds is necessary $[6,7]$. While this is mostly possible, even in the relevant range of parts per billion (ppb) with expensive and bulky analytical equipment, it remains a challenge for relatively inexpensive and small chemical sensors, especially in a non-lab environment. On the other hand, cheap and small devices are necessary to routinely equip buildings with 
automated ventilation and keep a healthy indoor environment.

This work aims at selective detection of formaldehyde, one of the most hazardous indoor pollutants [8], in a real-case scenario. We use a silicon carbide based field-effect transistor (SiCFET) [9] with temperature cycled operation (TCO) for improved sensitivity and selectivity. Its performance is evaluated using reference instruments for formaldehyde concentration, temperature, humidity, and $\mathrm{CO}_{2}$ concentration.

\section{Sensor}

A silicon carbide based field-effect transistor (SiC-FET, Fig. 3a) [9] is used as low-cost gas sensor. The transistor is made from 4" n-type $4 \mathrm{H}-\mathrm{SiC}$ wafers. The transistor structure's gate electrode is open to the environment and made out of a catalytic material, in this case $25 \mathrm{~nm}$ of porous iridium (Ir) deposited by room temperature DC magnetron sputtering at an argon pressure of 50 mTorr. The gate oxide is tungsten trioxide $\left(\mathrm{WO}_{3}\right)$ deposited by PLD (pulsed laser deposition). This combination of materials has already shown promising selectivity towards VOCs like naphthalene [10], and good sensitivity to formaldehyde (Fig. 1b). The sensor can be heated up to $400{ }^{\circ} \mathrm{C}$ with an external ceramic heater. The temperature is measured with a Pt-100, but not controlled to enable quick steps for the TCO (Fig. 2a).

\section{Field test system}

The field test system comprises a SiC-FET sensor with appropriate electronics for control and read-out. The electronics is controlled with a LabVIEW program running on an Intel compute stick which drives the SiC-FET and

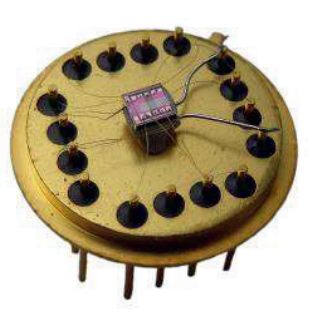

(a)

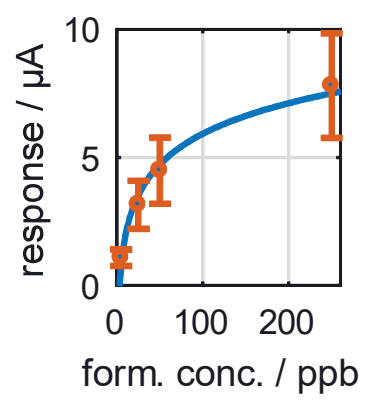

(b)

Fig. 1: (a) A SiC-FET sensor chip mounted to a TO-8 header together with a Pt100 temperature sensor. (b) Logarithmic sensor response dependent on formaldehyde concentration.

logs its data as well as the $\mathrm{CO}_{2}$ concentration $\left(\mathrm{C}_{\mathrm{CO} 2}\right)$ from a reference system (SenseAir tSense). Remote access is possible via an external $4 G$ modem.

\section{Operating mode and data processing}

Formaldehyde concentration ( $\mathrm{c}_{\text {Form }}$ ), ambient temperature $(T)$, and relative humidity $(\mathrm{RH})$ are measured and recorded with a GrayWolf FM801 [11] with a measurement range between 10 and $1000 \mathrm{ppb}$ and very low cross-sensitivity to other gases. It produces one measurement every $30 \mathrm{~min}$.

Temperature cycled operation (TCO) is often used to enhance sensitivity and selectivity of chemical sensors [12]. Reactivity and/or the reaction path of chemicals on the sensor surface depend strongly on surface temperature and, thus, a change in temperature effectively changes the sensor's behavior. The resulting data is similar to data that could be obtained from a sensor array with many different sensors, but often with reduced drift

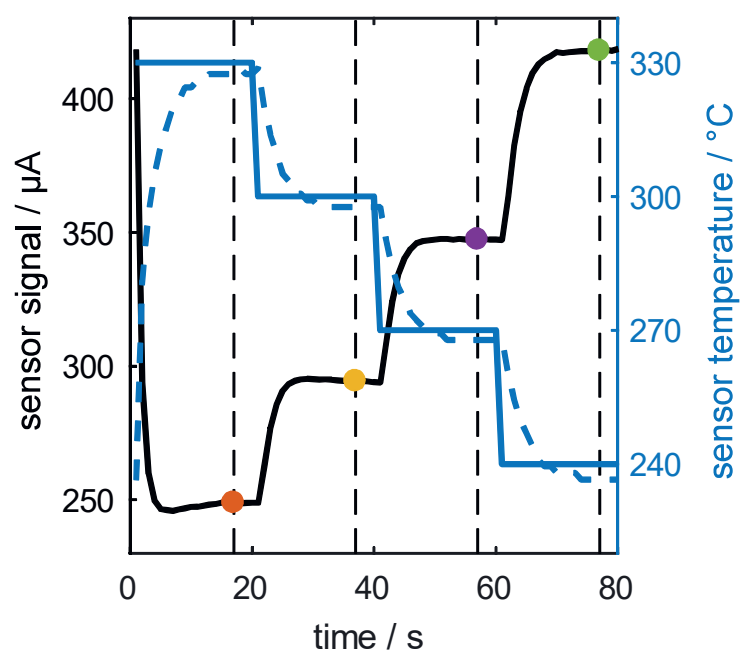

(a)

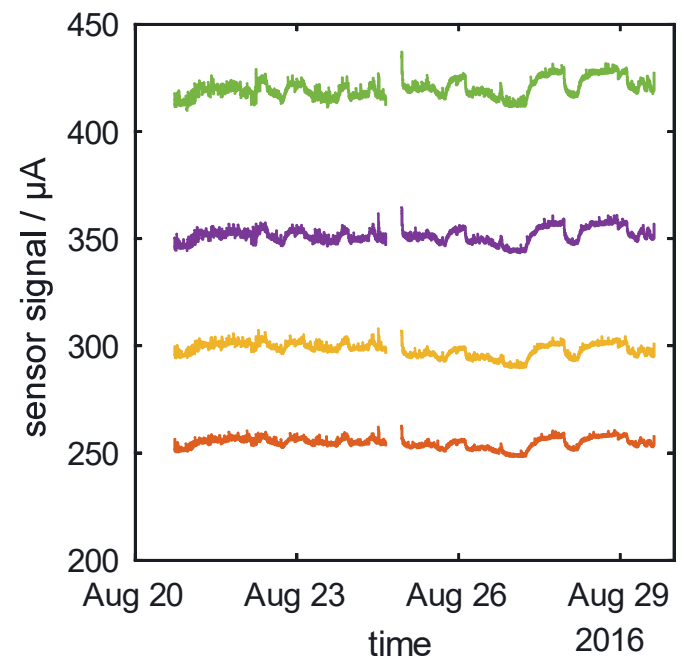

(b)

Fig. 2: (a) Temperature cycle (blue) and resulting sensor signal. The middle of each period of time, from which the mean is computed as virtual sensor, is marked with a colored dot. (b) Virtual sensors extracted from the sensor signal. 
and less chance of failure. A drawback is the longer measurement time since the data is produced sequentially and not, as with a real sensor array, in parallel.

In this work, the sensor is heated to 330,300 , 270 , and $240{ }^{\circ} \mathrm{C}$ for $20 \mathrm{~s}$ each, i.e. one temperature cycle takes $80 \mathrm{~s}$ to complete (Fig. 2a). The sensor signal is then evaluated at the end of each temperature plateau; in this case, with a sample rate of $1 \mathrm{~s}$, we compute the mean of the last six points of each plateau, resulting in four virtual sensors (Fig. 2b).

All reference data $\left(\mathrm{c}_{\mathrm{Form}}, \mathrm{T}, \mathrm{RH}, \mathrm{c}_{\mathrm{CO} 2}\right.$ ) are linearly interpolated to a sampling rate of $80 \mathrm{~s}$ to match the gas sensor data. We compute the Pearson correlation between the reference data and the four virtual sensors as well as the principal components of the sensor data computed with principal component analysis (PCA). To compute real concentrations from the sensor data, we also train and validate a multivariate regression model based on partial least squares regression (PLSR). The best of the resulting twelve sensor signals are then ranked using the TOPSIS (Technique for Order Preference by Similarity to Ideal Solution) [13] algorithm for best correlation with the $\mathrm{C}_{\text {Form }}$ reference.

\section{Results and Discussion}

We consider nine days of continuous (except for one short pause) data collection from the gas sensor and all reference systems $(9,287$ data points per system after interpolation). Tab. 1 shows the correlation between the variables ( $\mathrm{c}_{\text {Form }}, \mathrm{T}, \mathrm{RH}, \mathrm{C}_{\mathrm{CO} 2}$ ) themselves. Tab. 2 shows the correlation between the twelve extracted sensor signals and these four variables, together with the relative closeness to the optimal solution computed by TOPSIS.

Remarkably, $\mathrm{C}_{\mathrm{Form}}$ is negatively correlated to both $\mathrm{T}$ and $\mathrm{RH}$, which is opposite to the usually observed behavior $[14,15]$. However, the inverse correlation between $\mathrm{T}$ and $\mathrm{RH}$, i.e. $\mathrm{RH}$ decreases with increasing $T$, as well as the plausible variation over the day (Fig. 3) for both values indicates valid measurements. We concluded that this inverse correlation is mediated by the pattern superimposed from both the day/night cycle and the ventilation pattern. This conclusion is supported by the strong negative correlation between $\mathrm{C}_{\mathrm{Form}}$ and $\mathrm{C}_{\mathrm{CO} 2}$ : the reference instrument is not influenced by $\mathrm{CO}_{2}$ or other gases which are, here, only produced by humans in significant amounts and, thus, only during working hours, whereas formaldehyde builds up only at night and weekends while the ventilation is switched off, due to emission from building materials and/or
Tab. 1: Correlations between the four measured variables.

\begin{tabular}{|c|c|c|c|c|}
\hline & 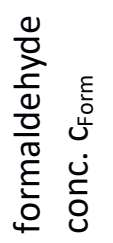 & 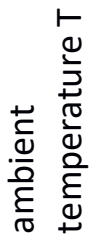 & 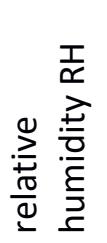 & 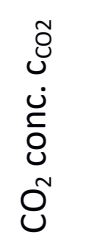 \\
\hline $\mathrm{C}_{\text {Form }}$ & 1.00 & -0.14 & -0.12 & -0.44 \\
\hline $\mathrm{T}$. & & 1.00 & -0.26 & -0.15 \\
\hline $\mathrm{RH}$ & & & 1.00 & 0.32 \\
\hline $\mathrm{c}_{\mathrm{CO} 2}$ & & & & 1.00 \\
\hline
\end{tabular}

Tab. 2: Pearson correlation of virtual sensors (Txxx) at different sensor temperatures, principal components (PCX), and PLSR with a varying number of components (PLSRX), with the four variables investigated. The relative closeness is computed by the TOPSIS algorithm, based on the best fit to the correlation pattern for the formaldehyde reference instrument.

\begin{tabular}{|c|c|c|c|c|c|}
\hline $\begin{array}{l}\frac{\bar{\pi}}{\bar{c}} \\
\frac{\sqrt[000]{n}}{2}\end{array}$ & 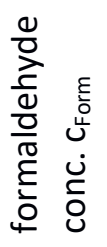 & 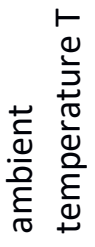 & 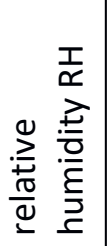 & 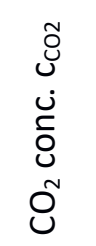 & 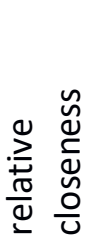 \\
\hline T330 & 0.46 & -0.22 & -0.06 & 0.04 & 0.75 \\
\hline T300 & 0.49 & -0.32 & -0.06 & 0.13 & 0.53 \\
\hline T270 & 0.46 & -0.08 & -0.03 & -0.10 & 0.80 \\
\hline T240 & 0.40 & 0.02 & -0.03 & $-0.1 \varepsilon$ & 0.61 \\
\hline PC1 & 0.47 & -0.11 & -0.04 & -0.08 & 0.87 \\
\hline PC2 & 0.15 & -0.40 & -0.04 & 0.35 & 0.30 \\
\hline PC3 & -0.03 & 0.08 & 0.03 & -0.01 & 0.30 \\
\hline PC4 & -0.16 & 0.05 & -0.06 & 0.00 & 0.47 \\
\hline PLSR1 & 0.48 & -0.13 & -0.04 & -0.06 & 0.88 \\
\hline PLSR2 & 0.50 & -0.23 & -0.05 & 0.04 & 0.71 \\
\hline PLSR3 & 0.52 & -0.24 & -0.03 & 0.04 & 0.68 \\
\hline PLSR4 & 0.52 & -0.24 & -0.03 & 0.04 & 0.69 \\
\hline
\end{tabular}

furniture (Fig. 3). This leads to a somewhat inverse behavior of $\mathrm{C}_{\mathrm{Form}}$, peaking during nights, and $\mathrm{c}_{\mathrm{CO} 2}$, peaking during days, which eventually results in a negative correlation of these two variables.

These findings must be taken into account when employing TOPSIS to find the bestperforming model. Usually, one would strive for high correlation to $\mathrm{C}_{\text {Form }}$ and low correlation to everything else, which is the expected result for the formaldehyde reference measurement. Instead, as mentioned previously, the reference shows a strong negative correlation especially 


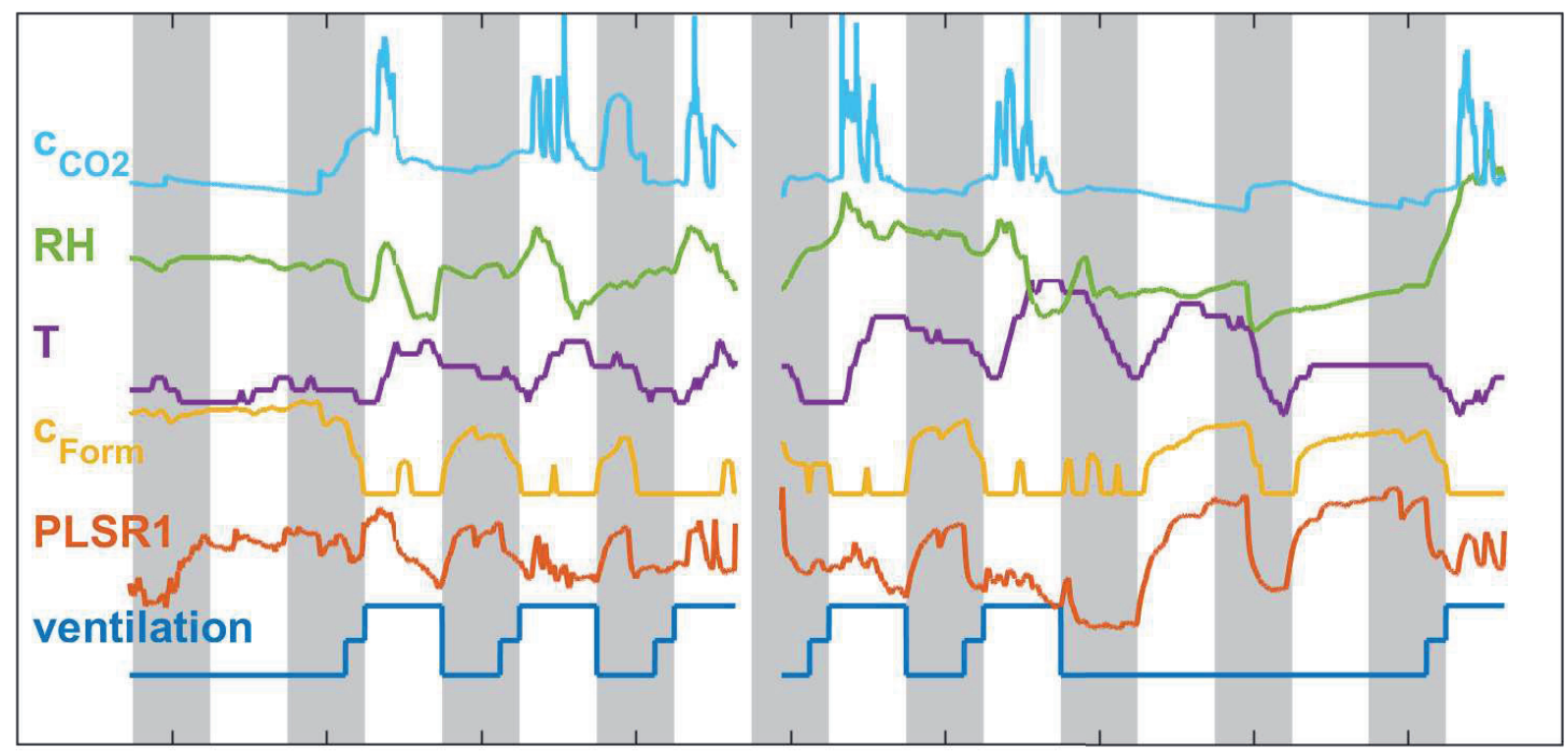

Aug 21 Aug 22 Aug 23 Aug 24 Aug 25 Aug 26 Aug 27 Aug 28 Aug 29

Fig. 3: Representation of all signals over the course of the measurement, standardized and shifted for visualization. PLSR1 is computed from virtual sensors and smoothed with a window size of 22 ( 30 min) for visualization; ventilation represents the usual, automated ventilation schedule of the school without respect to possibly unplanned events. A mark on the x-axis represents the start of the respective day, i.e. midnight, and darker areas represent night from 6pm to 6am. Aug 20/21 and Aug 27/28 were weekends (no school), and the short pause during Aug 24 is due to incomplete data streams during that time.

with $\mathrm{CO}_{2}$ as a result of the day/night and ventilation cycles. Hence, a sensitive and selective sensor should be able to replicate the reference's correlation pattern, and any differences would, in this case, indicate crosssensitivity. In Fig. 3 for instance, PLSR1 reacts to both $\mathrm{C}_{\text {Form }}$ and $\mathrm{C}_{\mathrm{CO} 2}$, i.e. it shows crosssensitivity to $\mathrm{CO}_{2}$, which manifests as a much higher correlation $(-0.06, \mathrm{Tab} .2)$ compared to the reference $(-0.44, \mathrm{Tab} .1)$. Therefore, the relative closeness was computed not with the actual correlations, but with the absolute difference of correlations in Tab. 2 and the correlations in the first row of Tab. 1, effectively searching for the best match with the correlation pattern observed for the reference. All variables were weighted equally.

The relative closeness of each signal is displayed in the right column of Tab. 2; higher values are better. It can be seen that both data processing methods (PCA and PLSR) improve the result compared with the best virtual sensor (T270) alone, and lead to more stable correlations with $\mathrm{T}$ and $\mathrm{RH}$ compared to what is observed for the virtual sensors. Both methods produce almost equally good results, which is remarkable because PLSR as supervised method could be expected to yield a better fit to the reference data. PC2 captures mostly the influence of ambient temperature, while PC3 and PC4 represent only noise. PLSR with more than one component increases correlation with
$\mathrm{C}_{\mathrm{Form}}$ slightly, but at the cost of much higher ambient temperature dependency.

It should be noted that the significant negative correlation between $\mathrm{C}_{\mathrm{CO} 2}$ and $\mathrm{C}_{\mathrm{Form}}(-0.44)$, as observed from the reference instrument signals, is never reached with any of the virtual sensor signals - the closest value is 0.18 . Since the reference has no or very low cross-sensitivity to other gases, this indicates cross-sensitivity of the SiC-FET sensor to a gas correlated with $\mathrm{C}_{\mathrm{CO} 2}$. This is observed as peaks during the day which follow the shape of the $\mathrm{CO}_{2}$ peaks in Fig. 3. Due to its sensing principle a SiC-FET is, like most chemical sensors, not sensitive to $\mathrm{CO}_{2}$. However, the reaction could arise from other compounds correlated with human activity. Likely candidates are acetone or isoprene $[16,17]$ which are contained in human breath at $4 \%$ and $2 \%$ of $\mathrm{CO}_{2}$ concentration [17]. The mean peak height for $\mathrm{CO}_{2}$ is approximately $700 \mathrm{ppm}$, compared to a background level of $400 \mathrm{ppm}$, which then relates to an addition of $300 \mathrm{ppm} \mathrm{CO}_{2}$ through human breath, i.e. $12 \mathrm{ppm}$ acetone and $6 \mathrm{ppm}$ isoprene in the air. These concentrations are, in sum, roughly 500 times higher than the maximum formaldehyde concentration (30-35 ppb) and produce a sensor signal similar to $10-15 \mathrm{ppb}$ formaldehyde. Hence, while the selectivity still has to be improved for this use-case, the sensor currently shows a very good selectivity of about $50: 1$ to formaldehyde vs. breath 
components assuming that formaldehyde is the main component accumulating. Indeed, many studies show that formaldehyde is most prevalent of all VOCs in indoor air [18-20]. Other compounds often found, however in lower concentrations, are acetaldehyde, hexanal, toluene, and a-pinene, among others. The changes in sensor signal in this field test were about twice as high as could be expected from static sensitivity measurements (Fig. 1). This corresponds to a selectivity of $25: 1$, and shows that the sensor must be checked for cross-sensitivity with the mentioned compounds in future experiments.

Fig. 3 shows the course of all recorded signals, the computed PLSR1 (smoothed), and the automated ventilation schedule of the school, which does not account for manual overrides like, presumably, on Aug 28. The $5 \%$ and $95 \%$ percentiles are $[23.0 ; 27.0]{ }^{\circ} \mathrm{C}$ for ambient temperature $\mathrm{T}, \quad[40.4 ; 53.4] \%$ for relative humidity $\mathrm{RH}$, and $[335 ; 500] \mathrm{ppm}$ for $\mathrm{CO}_{2}$ concentration $\mathrm{C}_{\mathrm{CO} 2}$. $\mathrm{T}$ and $\mathrm{RH}$ show mostly opposite trends as expected, and $\mathrm{C}_{\mathrm{CO} 2}$ seems to be a valid indicator for human presence as it only appears during working days and in sharp peaks which reflect classes and breaks. All values of $\mathrm{c}_{\text {Form }}$ measured with the reference instrument below $10 \mathrm{ppb}$ were set to zero due to the instrument's limited measurement range; the highest peak was $34 \mathrm{ppb}$ for the reference (Aug 21) and 24.5 ppb for PLSR1 (Aug 28). The ventilation was off during most of the night and at weekends, set to $50 \%$ at 3 am, and to $100 \%$ from $6 a m$ to $6 \mathrm{pm}$. This pattern is to a good portion reflected in the formaldehyde concentration (both reference and PLSR1), as it increases with ventilation shut off and rapidly decreases when ventilation is switched on. However, all in all, the measured concentrations are far below the recommended threshold limit value of $80 \mathrm{ppb}$ for formaldehyde [8].

\section{Conclusion}

We have investigated a SiC-FET with $\mathrm{Ir} / \mathrm{WO}_{3}$ as sensitive material as an inexpensive and small device to selectively measure formaldehyde in the ppb range under real-world conditions. The test site was a school equipped with a ventilation system. Temperature cycled operation and multivariate data processing was employed to improve the correlation between sensor signal and reference instrument. Temperature, relative humidity, and $\mathrm{CO}_{2}$ concentration were also recorded. The evaluation shows a distinct correlation pattern for the reference instrument with the other recorded variables arising from the day/night cycle. Taking this into account, the first principal component and a PLS regression with one component produce almost equally good results with a correlation to the reference instrument of $0.47 / 0.48$. A cross-correlation to an unidentified component of, presumably, human breath is seen, with a selectivity ratio of $25: 1$ towards formaldehyde.

We will now validate this data with controlled measurements in the lab, targeting especially cross-sensitivity towards other VOCs commonly found in indoor air and working towards reliable formaldehyde detection in a changing VOC background.

\section{Acknowledgments}

The authors would like to thank Montessoriskolan Trilobiten (Linköping, Sweden) for providing the premises to conduct the field tests.

This project has received funding from the European Union's Seventh Framework Programme for research, technological development and demonstration under grant agreement No 604311.

\section{References}

[1] EU, Directive 2010/31/EU of the European Parliament and of the Council of 19 May 2010 on the energy performance of buildings (recast), Off. J. Eur. Union. (2010) 13-35. doi:doi:10.3000/17252555.L_2010.153.eng.

[2] M. Kraus, D. Kubečková, Airtightness of Energy Efficient Buildings, GSTF J. Eng. Technol. 2 (2013) 14-20. doi:10.5176/2251-3701_2.2.74.

[3] N.E. Klepeis, W.C. Nelson, W.R. Ott, J.P. Robinson, A.M. Tsang, P. Switzer, et al., The National Human Activity Pattern Survey (NHAPS): a resource for assessing exposure to environmental pollutants., J. Expo. Anal. Environ. Epidemiol. 11 (2001) 231-252. doi:10.1038/sj.jea.7500165.

[4] U. Satish, M.J. Mendell, K. Shekhar, T. Hotchi, D. Sullivan, S. Streufert, et al., Is $\mathrm{CO} 2$ an indoor pollutant? direct effects of low-to-moderate $\mathrm{CO} 2$ concentrations on human decision-making performance, Environ. Health Perspect. 120 (2012) 1671-1677. doi:10.1289/ehp.1104789.

[5] L. Mølhave, G.D. Nielsen, Interpretation and limitations of the concept "Total volatile organic compounds"(TVOC) as an indicator of human responses to exposures of volatile organic compounds (VOC) in indoor air, Indoor Air. 2 (1992) 65-77.

[6] L. Mølhave, B. Bach, O.F. Pedersen, Human reactions to low concentrations of volatile organic compounds, Environ. Int. 12 (1986) 167-175. doi:10.1016/0160-4120(86)90027-9.

[7] B. Seifert, Richtwerte für die Innenraumluft: Die Beurteilung der Innenraumlufqualität mit Hilfe der Summe der flüchtigen organischen Verbindungen 
(TVOC-Wert), Bundesgesundheitsblatt Gesundheitsforsch. - Gesundheitsschutz. 42 (1999) 270-278.

[8] K. Koistinen, D. Kotzias, S. Kephalopoulos, C. Schlitt, P. Carrer, M. Jantunen, et al., The INDEX project: Executive summary of a European Union project on indoor air pollutants, Allergy Eur. J. Allergy Clin. Immunol. 63 (2008) 810-819. doi:10.1111/j.1398-9995.2008.01740.x.

[9] M. Andersson, R. Pearce, A.L. Spetz, New generation SiC based field effect transistor gas sensors, Sensors Actuators, B Chem. 179 (2013) 95-106. doi:10.1016/j.snb.2012.12.059.

[10] M. Bastuck, D. Puglisi, J. Huotari, T. Sauerwald, J. Lappalainen, A.L. Spetz, et al., Exploring the selectivity of WO 3 with iridium catalyst in an ethanol / naphthalene mixture using multivariate statistics, Thin Solid Films. 618 (2016) 263-270. doi:10.1016/j.tsf.2016.08.002.

[11] Graywolf Sensing Solutions, Graywolf FM-801 Datasheet, (n.d.). https://www.wolfsense.com/pdf/GrayWolfFormaldehyde_Monitor_brochure-1011.pdf (accessed March 31, 2017).

[12] P. Reimann, A. Schütze, Sensor Arrays, Virtual Multisensors, Data Fusion, and Gas Sensor Data Evaluation, in: C.-D. Kohl, T. Wagner (Eds.), Gas Sens. Fundam., Springer Berlin Heidelberg, Berlin, Heidelberg, 2014: pp. 67-107. doi:10.1007/978-3-642-54519-1.

[13] C.-L. Hwang, K. Yoon, Multiple Attribute Decision Making, 186 (1981). doi:10.1007/978-3-64248318-9.

[14] T. Salthammer, S. Mentese, R. Marutzky, Formaldehyde in the indoor environment, Chem. Rev. 110 (2010) 2536-2572. doi:10.1021/cr800399g.

[15] A. Blondel, H. Plaisance, Screening of formaldehyde indoor sources and quantification of their emission using a passive sampler, Build. Environ. 46 (2011) 1284-1291. doi:10.1016/j.buildenv.2010.12.011.

[16] J. Kwak, B.A. Geier, M. Fan, S.A. Gogate, S.A. Rinehardt, B.S. Watts, et al., Detection of volatile organic compounds indicative of human presence in the air, J. Sep. Sci. 38 (2015) 2463-2469. doi:10.1002/jssc.201500261.

[17] P.R. Veres, P. Faber, F. Drewnick, J. Lelieveld, J. Williams, Anthropogenic sources of VOC in a football stadium: Assessing human emissions in the atmosphere, Atmos. Environ. 77 (2013) 1052-1059. doi:10.1016/j.atmosenv.2013.05.076.

[18] H. Schleibinger, U. Hott, D. Marchl, P. Braun, P. Plieninger, $\mathrm{H}$. Rüden, VOC-concentrations in Berlin indoor environments between 1988 and 1999, Gefahrstoffe - Reinhaltung Der Luft. (2001) 26.

[19] H. Neumann, M. Buxtrup, S. Benitez, J. Hahn, VOC- und Aldehydkonzentrationen in beschwerdefreien Klassenräumen unter unterschiedlichen Nutzungs- und Lüftungsbedingungen, 74 (2014) 85-94.

[20] B. Clarisse, A.M. Laurent, N. Seta, Y. Le Moullec, A. El Hasnaoui, I. Momas, Indoor aldehydes: Measurement of contamination levels and identification of their determinants in Paris dwellings, Environ. Res. 92 (2003) 245-253. doi:10.1016/S0013-9351(03)00039-2. 